\title{
ADVERSE EVENTS OF ANTIPSYCHOTICS AND CYTOCHROME POLYMORPHISMS: A CASE SERIES ON 31 PATIENTS
}

\author{
Giuseppe Maina ${ }^{1,2}$, Stefano Bramante ${ }^{1,2}$, Antonio Borsotti ${ }^{1,2}$, Francesco Oliva $^{2,3}$, \\ Sylvia Rigardetto ${ }^{2} \&$ Umberto Albert ${ }^{4,5}$ \\ ${ }^{1}$ Rita Levi Montalcini Department of Neurosciences, University of Turin, Turin, Italy \\ ${ }^{2}$ San Luigi Gonzaga University Hospital, Turin, Italy \\ ${ }^{3}$ Department of Clinical and Biological Sciences, University of Turin, Turin, Italy \\ ${ }^{4}$ Department of Medicine, Surgery and Health Sciences, University of Trieste, Trieste, Italy \\ ${ }^{5}$ Department of Mental Health, Psychiatric Clinic, Azienda Sanitaria Universitaria Giuliano-Isontina, Trieste, Italy
}

received: 13.6.2021;

revised: 11.8.2021;

accepted: 5.9 .2021

\section{SUMMARY}

Background: Adverse events (AEs) contribute to poor outcome in patients affected by mental disorders. The aim of this case series is to describe how many antipsychotics-associated serious AEs could have been prevented if we had known in advance the genetic profile of the patient.

Subjects and methods: Data of patients who required the prescription of an antipsychotic drug, with a history of a documented antipsychotics-associated serious $A E$ and who underwent Neuropharmagen ${ }^{\circledR}$ test were retrospectively collected.

Results: Thirty-three subjects were selected for eligibility; two of them were excluded. Twelve subjects (38.7\%) showed genetic polymorphisms most likely associated to an increased risk of AE, with pharmacokinetic variations being the most prevalent. Moreover, the $35.5 \%$ of the total sample revealed drug-drug interactions (pharmacodynamic/pharmacokinetic) associated with increased risk of $A E$.

Conclusions: This case series highlights how pharmacogenetics testing with decision support tools, if applied earlier during the treatment with antipsychotics, could have led to identifying individuals at risk for serious AEs.

Key words: adverse events - antipsychotics - neuropharmagen - pharmacogenetics - cytochromes

$$
* * * * *
$$

\section{INTRODUCTION}

Psychiatric disorders are highly prevalent among the general population, contribute to a significant proportion of disease burden worldwide and are the leading cause of years lived with disability among all disease groups (Whiteford et al. 2015; Whiteford et al. 2013; Wittchen et al. 2011). Furthermore, mental disorders have significant economic direct and indirect costs that exceed those associated with other chronic medical conditions, such as diabetes and arterial hypertension (Soni 2011).

Several factors can contribute to poor outcome and increasing costs associated with mental disorders; among them, sub-optimal response to available therapies and the co-occurrence of adverse events (AEs), which severely impact treatment adherence, may play a predominant role. Current psychiatric pharmacotherapy is indeed mainly based on a trial-and-error approach: as a consequence, many patients are exposed to several subsequent trials before responding to medications, spending months-to-years with severe and disabling symptoms (Mrazek 2010).

Pharmacogenetics testing, therapeutic drug monitoring and biomarkers are among the most promising approaches for applying a personalized intervention to mental disorders. According to the National Human Genome Research Institute, pharmacogenetics combines the science of how drugs work (pharmacology), with the science of the human genome (genomics), in order to understand how genetic variability influences drug pharmacokinetics/pharmacodynamics and treatment outcomes. This variability has relevance for dosing, therapeutic sensitivity, likelihood of side-effects, risk for hypersensitivity reactions and can partially explain the different response and tolerability profile associated with pharmacological therapies. Knowing a patient's genetic profile could be a useful resource in order to develop more personalized therapeutic interventions (Drozda et al. 2014; Gardner et al. 2014; Winner et al. 2015).

The role of individual gene variants in the metabolism and response to psychotropic medications has been mostly studied with regard to antidepressants, and guidelines for the selection and/or dosing of tricyclic antidepressants and selective serotonin reuptake inhibitors have been recently published (DPWG; Hicks et al. 2015, 2017). A recent meta-analysis of randomized controlled trials in Major Depressive Disorder (MDD) found a significantly higher remission rate among patients who received pharmacogenetics informed treatment compared to treatment as usual, with greater benefit in patients who failed to tolerate or respond to at least one prior antidepressant trial (Bousman et al. 2019). Less attention has been devoted to the role of pharmacogenetics testing in predicting response or tolerability to antipsychotics (van Westrhenen et al. 2020; Yoshida \& 
Muller 2020); antipsychotic dosing advice according to the DPWG are available for only aripiprazole, risperidone, haloperidol and zuclopenthixol.

Antipsychotics (APs), especially atypical ones, represent the treatment of choice for many psychiatric disorders and may play a role in the treatment of other mental conditions such as anxiety disorders, obsessivecompulsive disorder, and trauma-related disorders (Albert et al. 2016; Huhn et al. 2019). However, in a significant portion of patients these drugs cause AEs, which occur in a range from mild side effects, non-compromising quality of life, to serious adverse events (SAEs), which may result in hospitalization or be life threatening (e.g. Neuroleptic Malignant Syndrome) (Stroup et al. 2018).

Concerning the correlation between AP-induced AEs and pharmacogenetics, the main focus has been given to pharmacokinetics and in particular to the cytochrome P450 (CYP), a complex of liver enzymes that metabolize the majority of antipsychotic drugs, and to the study of its polymorphisms (Altar et al., 2013). Genetic variations in these cytochromes may compromise the enzyme activity, resulting in changes in AP plasma concentrations and, therefore, the likelihood of patients experiencing SAEs. In particular, multiple allelic combinations of CYP450 genes may result in different phenotypes, which can be schematically summarized in four different categories: Extensive Metaboliser (EM), with two functioning sets of the gene (wild type) and normal enzymatic activity; Ultrarapid Metabolizer (UM), with one or more sets of the CYP gene with similar or increased activity, resulting in a greater enzymatic function, with faster drug metabolism; Intermediate Metaboliser (IM), with one functional set of the gene and one without any activity, or with both sets having an intermediate activity; Poor Metaboliser (PM), with both of the genes copies having no activity. IM and PM subjects, considering their slower enzymatic activity, tend to have higher AP plasma concentrations, thus showing more frequently AEs (Altar et al. 2013).

Other studies have suggested the involvement of additional genetic abnormalities in different enzyme complexes (mTOR pathway) in the origin of specific AEs, such as extrapyramidal side effects (EPS) (Boloc et al. 2018; Mas et al., 2015).

In the last years different pharmacogenetics companies have developed Decision Support Tools (DSTs), such as CNSDose, Genecept, GeneSight, NeuroIDgenetix and Neuropharmagen, to help clinicians in the process of medication selection. These tools classify treatments into different categories, according to the patient's genetic profile, in order to select medications more likely to have better treatment outcomes. However, DTSs' utility in clinical practice has been rarely evaluated in large clinical trials and the available data are mainly focused on the role of these tools in predicting treatment response rather than AEs (Brown et al. 2020; Vilches et al. 2019). Regarding AEs, Bradely and colleagues (2018) did not find statistical differences between the experimental (pharmacogenetics NeuroIDgenetix guided) and the control group in a multicentre, prospective, randomized, subject and rater-blinded trial. AEs were collected using the Adverse Drug Event form and patients were treated with antidepressants, atypical antipsychotics and benzodiazepines (Bradely et al. 2018). Three other research groups studied the utility of Neuropharmagen in preventing the risk of AEs, highlighting how choosing a therapy in accordance with one's genetic profile lowered the probability of having AEs (Han et al., 2018; Ielmini et al., 2018; Perez et al., 2017). Two of them (Han et al., 2018; Perez et al., 2017) were restricted to individuals treated with antidepressants, while Ielmini and colleagues (2018) investigated subjects with bipolar disorder receiving mood stabilizers, antidepressants and/or antipsychotics. They showed that the group receiving a therapy modification concordant to the NFG test presented a significant reduction of AEs, evaluated through the Dosage Record and Treatment Emergent Symptom Scale (DOTES), after three months. No study, to our knowledge, specifically evaluated whether pharmacogenetics testing with NFG could prevent the occurrence of SAEs associated with the use of antipsychotics.

In clinical practice, often the use of pharmacogenetics testing is reserved to subjects having failed several AP trials or having experienced SAEs associated with APs. It remains to be evaluated whether using DSTs earlier in the treatment of subjects prescribed antipsychotics could prevent the occurrence of SAEs, resulting in less costs associated with new hospitalizations or prolonged hospitalizations.

The aim of this case series is to describe how many AP-associated SAEs could have been prevented if we had known in advance the genetic profile of the patient.

\section{SUBJECTS AND METHODS}

\section{Patients}

In this case series, we examined all medical records of patients admitted to the Psychiatric Inpatient Unit of the San Luigi Gonzaga Hospital, Orbassano (University of Turin, Italy), from April 2015 to June 2020, who met the following inclusion criteria:

- age $\geq 18$ years;

- a DSM-5 psychiatric disorder having required the prescription of an antipsychotic drug (AP);

- history of a documented serious adverse event (SAE) associated with the use of an AP; we considered a SAE (in accordance to the European Medicines Agency guidelines on good pharmacovigilance practices) an adverse reaction that was life-threatening, required in-patient hospitalization or prolongation of existing hospitalization, resulted in persistent or significant disability or incapacity, or was a congenital anomaly/birth defect; 
- underwent Pharmaco-Genetic Testing (PGT) with Neuropharmagen ${ }^{\circledR}$. PGT is routinely used in standard clinical practice in our Department when deemed necessary by the treating psychiatrist, with patients with a history of serious adverse events related to psychopharmacological medications. The purpose of this practice is to help clinicians select medications with better treatment outcomes.

- signed informed consent both for the execution of the Neuropharmagen ${ }^{\circledR}$ test ("Consenso informato per il test Neurofarmagen $\left.{ }^{\circledR} "\right)$ and for the treatment of personal data ("Informativa per il trattamento dei dati ai sensi del D.LGS. 196/2003 con manifestazione di consenso"). All subjects who present at our inpatient and outpatient service do sign a written informed consent (reviewed by our Ethical Committee) to have their clinical data potentially used for teaching and research purposes. For the purposes of the present case series, a specific request was made to our Ethical Committee (Comitato Etico Interaziendale A.O.U. San Luigi Gonzaga di Orbassano, Italy) in order to have access to clinical records of all patients who agreed and signed the abovementioned written informed consent; the protocol was reviewed and approved by the Ethical Committee ("Pratica 46/2020", "Deliberazione del direttore generale N. 310 del 05/6/20").

\section{Assessments and procedures}

Data were collected with a format that covered the following areas:

- Socio-demographic and clinical data: age, gender, ethnicity, marital and occupational status, education level, principal psychiatric diagnosis, psychiatric and/or medical comorbidities (co-occurring or lifetime) and smoke. All psychiatric disorder diagnoses are made, in our clinical routine, according to DSM5 criteria.

- History of pharmacological treatments: information regarding psychopharmacological and medical treatments (compound, daily dose) were specifically collected, with a particular focus on those taken at the time of the occurrence of the SAE.

- type and onset of the SAE: adverse events were coded referring to the Medical Dictionary for Regulatory Activities Terminology (MedDRA) preferred terms. We only included individuals for whom it was possible to trace the occurrence and onset of the $\mathrm{SAE}$, with respect to the new prescription and assumption of the AP, and for whom we were sure about treatment adherence. For the subjects already hospitalized medication compliance is routinely monitored, while for individuals with an SAE that required subsequent hospitalization, treatment adherence related to the medications taken before being admitted to the hospital is routinely double-checked with the patient and the caregivers.

\section{Genetic analysis}

Neuropharmagen ${ }^{\circledR}$ test was used for pharmacogenetic analysis (AB-Biotics SA, Barcelona, Spain). This test, through a genetic analysis carried out from a sample of saliva, studies about 30 genetic polymerphisms involved in the response and tolerability to 50 different drugs. In particular the Neuropharmagen ${ }^{\circledR}$ report is composed of 4 sections:

- Summary table of all drugs analysed. For each drug four different results can be obtained, according to a colour code: "standard/white", no genetic variation of relevance for the treatment has been detected; "green", higher probability of positive response and/or lower risk of adverse reactions; "yellow", need for specific dose monitoring and/or lower probability of positive response; "red", increased risk of different types of AE.

- Interpretation of the results and recommendations: drugs that, according to the test, could induce a different reaction than the average population (coloured box) are listed in detail, showing possible different polymorphisms that were uncovered.

- Additional genetic information: description of genes involved in the response and tolerability to drugs, where relevant variations were detected.

- Pharmacological interactions attachment: it contains a list of the main drugs inhibiting or inducing CYP450 enzymes.

\section{Statistical analysis}

Socio-demographic and clinical features of the patients were summarized as mean and standard deviation (SD) for continuous variables and frequency and percentage for categorical variables. We carried out all the statistical analyses using the Statistical Package for Social Sciences (Version 25.0, SPSS; SPSS Inc., Chicago, IL, USA).

Table 1. Socio-demographic characteristics of the total sample $(\mathrm{n}=31)$

\begin{tabular}{lc}
\hline Characteristics & Total sample $(\mathrm{n}=31)$ \\
\hline Gender, n (\%) & \\
$\quad$ male & $18(58.1)$ \\
$\quad$ female & $13(41.9)$ \\
Age (years), mean $\pm s d$ & $43.0 \pm 16.3$ \\
Education (years), mean $\pm \boldsymbol{s} \boldsymbol{d}$ & $10.4 \pm 3.4$ \\
Marital status, n (\%) & \\
single & $19(61.4)$ \\
married & $8(25.8)$ \\
widowed & $2(6.4)$ \\
divorced/separated & $2(6.4)$ \\
Employement, n $(\%)$ & \\
full time job/ part time job & $5(16.2)$ \\
housewife/student & $4(12.9)$ \\
retired & $4(12.9)$ \\
unemployed & $18(58.0)$ \\
\hline
\end{tabular}




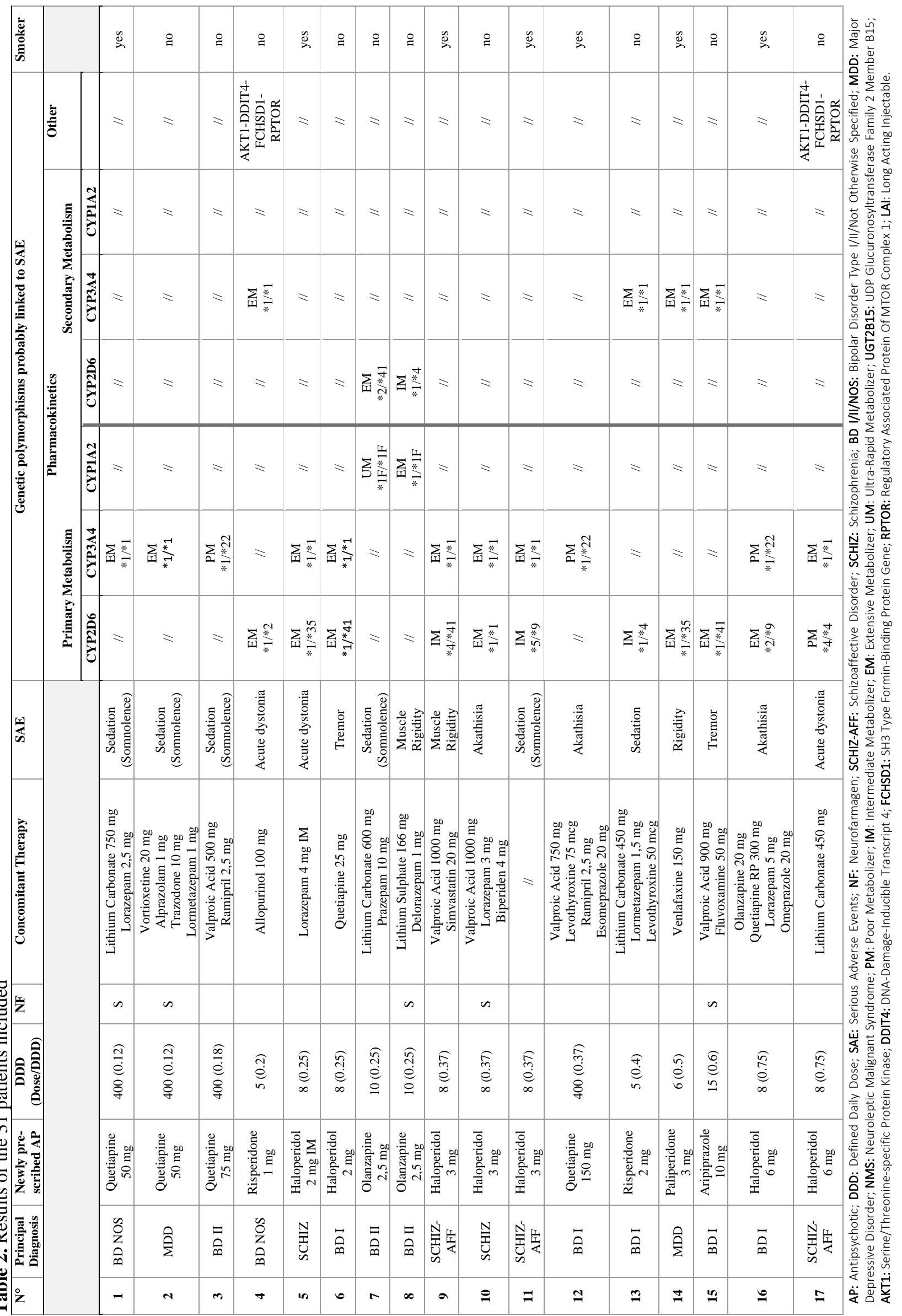




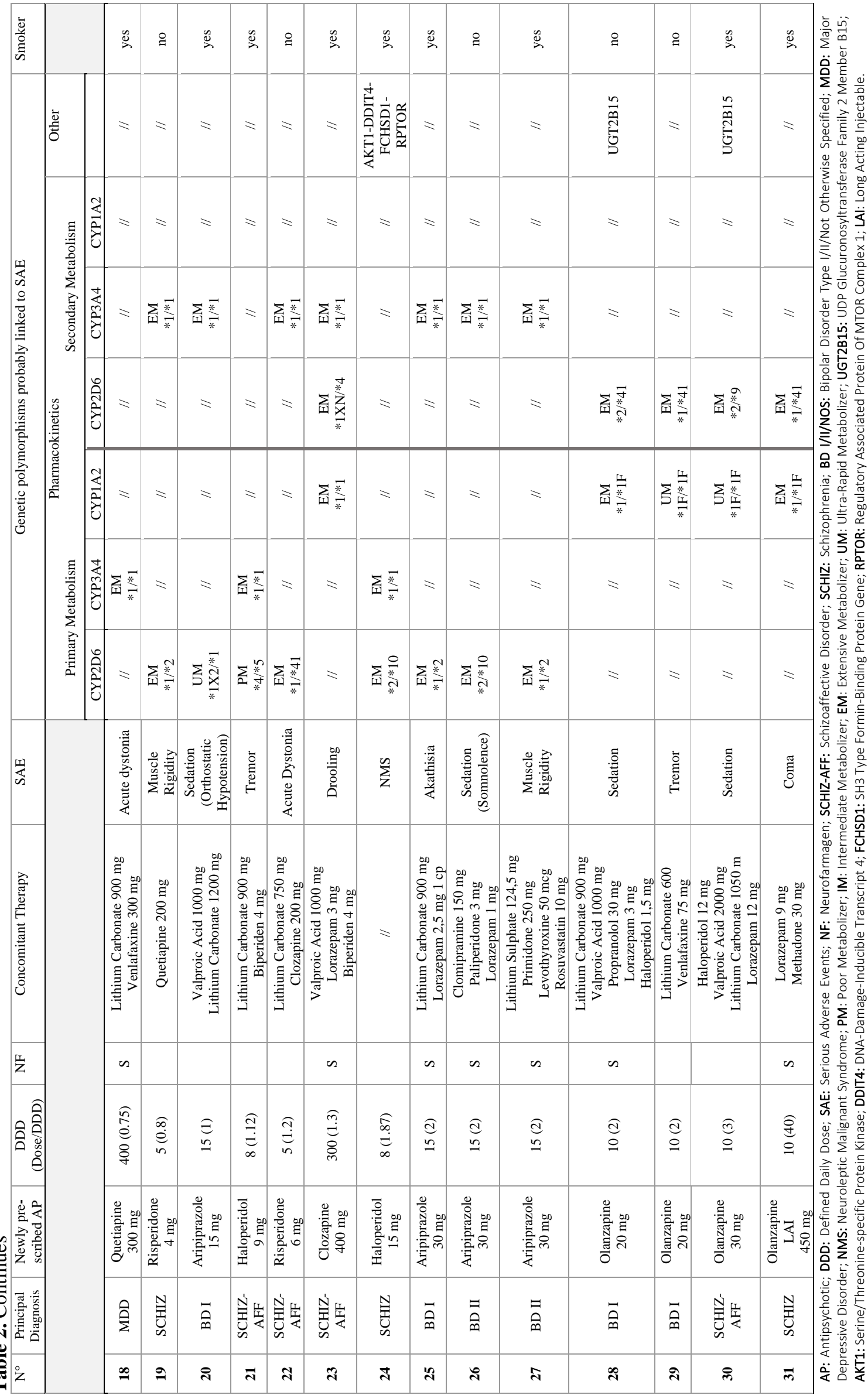




\section{RESULTS}

Thirty-three subjects were initially selected for eligibility, having a Neuropharmagen ${ }^{\circledR}$ test result; two of them were excluded because it was not possible to trace the onset of the SAE with regard to a new AP prescription, leaving 31 individuals. Sociodemographic characteristics of the sample are given in Table 1: all patients were Caucasian, $41.9 \%$ were females and the mean age of the sample was $43.0( \pm 16.3)$ years. Regarding medical comorbid disorders, three patients had hypertension, three hypothyroidism, two hypercholesterolemia, one obesity, one metabolic syndrome, one hyperuricemia, one chronic gastritis.

Table 2 provides specific information about antipsychotic treatments, concomitant therapies, serious adverse events and genetic polymorphisms possibly linked to SAEs for each patient. The majority of the sample (51.6\%) had a principal diagnosis of Bipolar Disorder, followed by Schizoaffective Disorder (22.6\%), Schizophrenia (16.1\%), and Major Depressive Disorder (9.7\%). Regarding the new AP possibly related to the occurrence/onset of the SAE, nine subjects $(29.0 \%)$ were prescribed Haloperidol, six (19.4\%) Olanzapine (one long-acting injectable Olanzapine), five (16.1\%) Aripiprazole, five (16.1\%) Quetiapine, four (13.0\%) Risperidone, one (3.2\%) Paliperidone and one (3.2\%) Clozapine. The most common AE was sedation $(n=10)$, followed by acute dystonia $(n=5)$, rigidity $(n=5)$, tremor $(n=4)$, akathisia $(n=4)$, drooling $(n=1)$, neuroleptic malignant syndrome $(\mathrm{n}=1)$ and coma $(n=1)$. All those AEs required inpatient hospitalization or prolongation of an existing hospitalization.

Concerning pharmacogenetics data (Table 2), 15 individuals (48.4\%) received a yellow (need for specific dose monitoring and/or lower probability of positive response) or red label (increased risk of different types of AE) associated with the newly prescribed antipsychotic (that associated with the occurrence of a SAE).

Twelve subjects $(38.7 \%)$ showed genetic polymerphisms most likely associated to an increased risk of developing AE. More specifically, eight subjects had exclusively polymorphisms of the cytochrome P450 enzyme drug-metabolizing system (primary and secondary metabolism), related to a decreased drug metabolism and an increased probability of higher blood levels of AP: four patients resulted IM phenotype (patient number 8, 9, 11 and 13) and four PM phenotype (patient number 3, 12, 16 and 17). Three individuals showed other genetic alterations: one subject (patient number 4) had polymorphisms of the mTOR pathway (AKT1DDIT4-FCHSD1-RPTOR), correlated to a major risk of EPS induced by AP, and two individuals (patient number 28 and 30) with UDP-glucuronosyltransferase 2B15 polymorphisms, associated to a reduction of Lorazepam elimination and to a greater risk of sedation. Moreover, one subject (patient number 17) had two combined genetic variations probably linked to SAE:
CYP2D6 PM phenotype plus polymorphisms of the mTOR pathway (AKT1-DDIT4-FCHSD1-RPTOR). In addition, patient number 24 did not show specific genetic alterations associated to neuroleptic malignant syndrome, however he was found to have AKT1DDIT4-FCHSD1-RPTOR polymorphism correlated to a major risk of EPS induced by AP, without experiencing such side effects.

Furthermore, regarding therapies taken together with AP treatment ("concomitant therapy") (Table 2), the $35.5 \%$ of the total sample revealed drug-drug interactions (pharmacodynamic and pharmacokinetic) associated with increased risk of AE. Among these subjects, five subjects were receiving a second antipsychotic in addition to basal AP therapy (patient number 6, 19, 22, 28 and 30), three an antidepressant (patient number 14, 15 and 29), one a benzodiazepine (patient number 23), one two other antipsychotics (patient number 16) and one another antipsychotic plus an antidepressant (patient number 26). No drug-drug interactions have been found with non-psychopharmacological treatments.

Lastly, three subjects showed both genetic polymorphisms and drug-drug interactions related to a higher risk of AE (patient number 16, 28 and 30).

Patients were subsequently treated with a different AP from the one suspected to be related with the SAE. According to NFG test results, the AP was selected among those who were not flagged as red or yellow. No new SAEs were reported.

\section{CONCLUSIONS}

The aim of the present case series was to describe how many AP-associated SAEs could have been prevented if we had known in advance the genetic profile of the patient. To date, this is the first paper specifically evaluating the relationship between the occurrence of serious adverse events and pharmacogenetics polymerphisms potentially altering APs metabolism, assessed through the administration of DSTs.

More than one third of the sample (38.7\%) showed genetic polymorphisms probably linked to the occurrence of SAEs, with pharmacokinetic variations being the most prevalent. The majority of these genetic anomalies resulted in a decreased activity of the CYP450 enzymes: the CYP2D6 polymorphisms were the most common, followed by those related to the cytochrome CYP3A4; no CYP1A2 PM/IM phenotypes were detected.

Our findings are consistent with previous pharmacogenomics studies evaluating the relationship between CYP450 slow metabolizer status (PM and IM phenotypes) and AP-induced AEs, where EPS, such as tardive dyskinesia, were most commonly associated (Altar et al. 2013). However, the majority of these studies have focused on the CYP2D6 polymorphisms, while the association between CYP3A4 and CYP1A2 genetic alterations and AP-induced side effects in clinical samples have been rarely evaluated and need to be further 
investigated. Moreover, regarding pharmacokinetic genetic variations, in our sample two patients experienced sedation as SAE and showed UDP-glucuronosyltransferase $2 \mathrm{~B} 15$ polymorphisms, resulting in a reduction of Lorazepam elimination. UGT2B15 enzymes are responsible for the conjugation and subsequent removal of many compounds, such as some medications (e.g. Lorazepam). In a previous Korean study, Chung and colleagues found a significant association between UGT2B15 genetic polymorphisms and the pharmacokinetics of Lorazepam: the UGT2B $15 * 2 / * 2$ homozygous variant group showed $40 \%$ to $50 \%$ lower systemic clearance and metabolic activity than the UGT2B15*1/*1 wild-type group (Chung et al. 2005). Concerning non-pharmacokinetic polymorphisms, two patients had AKT1-DDIT4-FCHSD1-RPTOR polymerphisms related to a major risk of AP-induced EPS and one patient showed an association between this polymorphism and neuroleptic malignant syndrome. AKT1-DDIT4-FCHSD1-RPTOR was the first pharmacogenetics predictor model, developed analyzing genegene interactions in nine genes related to the mTOR pathway, that demonstrated a high predictive relationship between an individual's genotype and AP-induced EPS (Mas et al. 2015). However, subsequent research demonstrated that the results of this previous analysis correlated only modestly with successive studies on the same association. Applying different algorithms, the authors found a new pharmacogenetics predictor model that included four polymorphisms in only two genes: AKT1 and RPTOR (Boloc et al. 2018). To our knowledge this is the first paper to find an association between mTOR pathway polymorphisms and neuroleptic malignant syndrome; therefore, our findings deserve further investigations in future research.

When evaluating the relationship between pharmacogenomics markers and the occurrence of SAEs, some confounding factors are worthy of attention: ethnicity, cigarette smoking, concomitant therapies/supplements, medical comorbidities and some physical conditions, such as pregnancy, that could influence the pharmacokinetic/pharmacodynamic. Ethnicity is an important element to consider in pharmacogenetics research: genotype and frequency of polymorphisms may differ significantly among populations (Guttman et al. 2019; Huang et al. 2015). In our case series all patients were European Caucasians and therefore the sample was homogeneous in terms of ethnicity. Regarding the second point, cigarette smoking is a great inducer of CYP1A2 enzyme activity, resulting in significant lower plasma concentrations of some drugs (e.g. Olanzapine and Clozapine) in smokers compared with non-smokers, due to an acceleration of the liver metabolism. Considering the high prevalence of smoking among patients affected by psychiatric disorders (Quigley et al. 2019; Scott et al. 2018), it is important to evaluate this habit in order to clearly understand the relationship between pharmacogenetics polymorphisms and side effects.
About half of our sample was represented by smokers, but, among these, only three individuals (patient number 23,30 and 31) were in therapy with agents metabolized by CYP1A2 enzyme. Notwithstanding the consequent reduced AP plasma concentrations in such subjects, serious drooling was experienced by patient number 23 , and serious sedation by patient number 30 . The first case could be explained by drug interactions between Clozapine and Lorazepam that expose patients to an increased risk of hypersalivation, as shown in previous studies (Edge et al. 1997), while the second case may be due to UGT2B15 polymorphisms. About concomitant therapies/supplements, despite the indications given by international guidelines suggesting monotherapy as a gold standard in treating psychiatric disorders, polytherapy is a common practice in acute-phase patients and during cross-titration (Centorrino et al. 2005; Hatta et al. 2019). The principal reason to avoid concurrent medications is that polytherapy increases the risk of side effects, raising the likelihood of pharmacodynamic and pharmacokinetic interactions, mainly by induction and inhibition mechanisms of the CYP450 complex (Wijesinghe 2016). CYP2D6 has a low threshold for inhibition and concomitant therapies have the same effect on the activity of this enzyme as genetic polymorphisms have (Altar at al. 2013); while, concerning CYP3A4 and CYP1A2 metabolism, inhibitions and inductions by other drugs seem to have a greater part than pharmacogenetics (Altar et al. 2013). Moreover, some compounds act as enzyme substrates, without having specific induction or inhibition activity, and could decelerate the metabolism of some drugs through a competitive mechanism, with subsequent increased plasma concentrations (Pelkonen et al. 2008). In our paper, a significant proportion of patients with SAEs (about one third of the total sample) revealed drug-drug interactions (pharmacodynamic and pharmacokinetic) associated with a greater risk of side effects, suggesting how concomitant therapies should be carefully evaluated. None of the patients was taking supplements. Finally, some medical conditions, such as liver diseases, could expose individuals to a higher risk of AEs by altering adsorption, distribution, metabolism or excretion of some drugs (Villeneuve et al. 2004); however, no such significant medical illnesses or other physical conditions, such as pregnancy, were found in our sample.

Our case series presents several limitations. Considering the retrospective nature of the study, two aspects need to be considered: first of all, variables were evaluated retrospectively from medical records and are therefore dependent on the precision of clinicians' data collection; secondly we lack information regarding AP plasma concentrations - Therapeutic Drug Monitoring (TDM) - meaning that we are unable to determine whether CYP 450 genetic polymorphisms and drug interactions are certainly related to higher drug plasma concentrations or not. Data on cigarettes smoking were 
only available as a categorial variable; the mean number of cigarettes smoked per day would be a better information in order to clearly understand the impact of this habit on CYP1A2 enzyme activity. Lastly, all individuals in this paper were inpatients with a likely more severe course of illness, which often requires polytherapy (psychotropic and non-psychotropic agents). NFG color coding could be misleading when drug-drug interactions are taken into consideration: when a specific substance results as optimal for the subject it is flagged as green, without considering possible medication interactions.

In conclusion, this case series highlights how pharmacogenetics testing with DSTs, if applied earlier during the treatment with antipsychotics, could have led to identifying individuals at risk for SAEs, possibly preventing a third of SAEs. The retrospective nature of our data, however, needs replication in prospective, randomized controlled trials.

\section{Acknowledgements: None.}

\section{Conflict of interest: None to declare.}

\section{Contribution of individual authors:}

Giuseppe Maina: Designed the study, wrote the draft and approved the final manuscript.

Stefano Bramante \& Antonio Borsotti: Collected patients' data, managed the literature searches, analyzed the data and approved the final manuscript.

Francesco Oliva \& Sylvia Rigardetto: Collected patients' data, managed the literature searches and approved the final manuscript.

Umberto Albert: Designed the study, analyzed the data, wrote the draft and approved the final manuscript.

All authors provided approval for the final manuscript draft.

\section{References}

1. Albert U, Carmassi C, Cosci F, De Cori D, Di Nicola Marco, Ferrari $S$, et al. Role and clinical implications of atypical antipsychotics in anxiety disorders, obsessivecompulsive disorder, trauma-related, and somatic symptom disorders: a systematized review. Int Clin Psychopharmacol 2016; 31:249-258

2. Altar CA, Homberger J, Shewade A, Cruz V, Garrison J, Mrazek D. Clinical validity of cytochrome P450 metabolism and serotonin gene variants in psychiatric pharmacotherapy. Int Rev Psychiatry 2013; 25(5): 509-33

3. Boloc D, Gortat A, Cheng-Zhang JQ, Garcia-Cerro S, Rodriguez N, Parellada M, et al. Improving pharmacogenetic prediction of extrapyramidal symptoms induced by antipsychotics. Transl Psychiatry 2018; 8(1): 276

4. Bousman CA, Arandjelovic K, Mancuso SG, Eyre HA, Dunlop BW. Pharmacogenetic tests and depressive symptom remission: a meta-analysis of randomized controlled trials. Pharmacogenomics 2019; 20:37-47
5. Bradley P, Shiekh M, Mehra V, Vrbicky K, Layle S, Olson $M C$ et al. Improved efficacy with targeted pharmacogenetic-guided treatment of patients with depression and anxiety: A randomized clinical trial demonstrating clinical utility. J Psychiatr Res 2018; 96:100-107

6. Brown L, Vranjkovic O, Li J, Yu K, Al Habbab T, Johnson $H$, et al. The clinical utility of combinatorial pharmacogenomic testing for patients with depression: a metaanalysis. Pharmacogenomics 2020; 21:559-569

7. Centorrino F, Fogarty KV, Sani G, Salvatore P, Cincotta $S L$, Hennen $J$, et al. Use of combinations of antipsychotics: McLean Hospital inpatients, 2002. Hum Psychopharmacol 2005; 20:485-92

8. Chung JY, Cho JY, Yu KS, Kim JR, Jung HR, Lim KS, et al. Effect of the UGT2B15 genotype on the pharmacokinetics, pharmacodynamics, and drug interactions of intravenous lorazepam in healthy volunteers. Clin Pharmacol Ther 2005; 77:486-94

9. DPWG (Dutch Pharmacogenetics Working Group): dosing guidelines, 2008 (available at https://www.pharmgkb.org/ page/dpwg accessed June 15, 2020)

10. Drozda K, Müller DJ, Bishop JR. Pharmacogenomic testing for neuropsychiatric drugs: current status of drug labeling, guidelines for using genetic information, and test options. Pharmacotherapy 2014; 34: 166-84

11. Edge ST, Markowitz JS, Devane L. Clozapine Drug-Drug Interactions: A Review of the Literature. Human Psychopharmacology 1997; 12:5-20

12. Gardner KR, Brennan FX, Scott R, Lombard J. The potential utility of pharmacogenetic testing in psychiatry. Psychiatry J 2014; 730956

13. Guttman Y, Nudel A, Kerem Z. Polymorphism in Cytochrome P450 $3 A 4$ Is Ethnicity Related. Front Genet 2019; 19:224

14. Han C, Wang SM, Bahk WM, Lee SJ, Patkar AA, Masand PS, et al. A Pharmacogenomic-based Antidepressant Treatment for Patients with Major Depressive Disorder: Results from an 8-week, Randomized, Single-blinded Clinical Trial. Clin Psychopharmacol Neurosci 2018; 16:469-480

15. Hatta K, Hasegawa H, Imai A, Sudo Y, Morikawa F, Katayama $S$, et al. Real-world effectiveness of antipsychotic monotherapy and polytherapy in 1543 patients with acute-phase schizophrenia. Asian J Psychiatr 2019; 40:82-87

16. Hicks JK, Sangkuhl K, Swen JJ et al. Clinical pharmacogenetics implementation consortium guideline (CPIC) for CYP2D6 and CYP2C19 genotypes and dosing of tricyclic antidepressants: 2016 update. Clin Pharmacol Ther 2017; 102:37-44

17. Hicks, JK, Sangkuhl, K, Swen, JJ, Ellingrod VL, Muller DJ, Shimoda K, et al. Clinical Pharmacogenetics Implementation Consortium (CPIC) Guideline for CYP2D6 and CYP2C19 Genotypes and Dosing of Selective Serotonin Reuptake Inhibitors. Clinical Pharmacology and Therapy 2015; 98:127-134

18. Huang, T, Shu, Y, Cai, Y-D. Genetic differences among ethnic groups. BMC Genomics 2015; 16:1093

19. Huhn M, Nikolakopoulou A, Schneider-Thoma J, Krause $M$, Samara $M$, Peter $N$, et al. Comparative efficacy and tolerability of 32 oral antipsychotics for the acute treatment of adults with multi-episode schizophrenia: a systematic review and network meta-analysis. The Lancet 2019; 394(10202): 939-951 
20. Ielmini M, Poloni N, Caselli I, Espadaler J, Tuson M, Grecchi A, et al. The utility of pharmacogenetic testing to support the treatment of bipolar disorder. Pharmacogenomics Pers Med 2018; 11: 35-42

21. Mas S, Gassó P, Ritter MA, Malagelada C, Bernardo M, Lafuente A, et al. Pharmacogenetic predictor of extrapyramidal symptoms induced by antipsychotics: Multilocus interaction in the mTOR pathway. Eur Neuropsychopharmacol 2015; 25:51-59

22. Mrazek DA. Psychiatric pharmacogenomics testing in clinical practice. Dialogues Clin Neurosci 2010; 12:69-76 PMID: 20373668

23. Pelkonen O, Turpeinen M, Hakkola J, Honkakoski P, Hukkanen J, Hanno R. Inhibition and induction of human cytochrome P450 enzymes: current status. Arch Toxicol 2008; 82(10): 667-715

24. Pérez V, Salavert A, Espadaler J, Tuson M, Saiz-Ruiz J, Saez-Navarro C, et al. Efficacy of prospective pharmacogenetic testing in the treatment of major depressive disorder: results of a randomized,double-blind clinical trial. BMC Psychiatry 2017; 14;17(1): 250

25. Quigley H, MacCabe JH. The relationship between nicotine and psychosis. Ther Adv Psychopharmacol 2019; 1:9-12

26. Scott JG, Matuschka L, Niemelä S, Miettunen J, Emmerson B, Mustonen A. Evidence of a Causal Relationship Between Smoking Tobacco and Schizophrenia Spectrum Disorders. Front Psychiatry 2018; 20:607

27. Soni A. Top 10 Most Costly Conditions among Men and Women, 2008: Estimates for the U.S. Civilian Noninstitutionalized Adult Population, Age 18 and Older. Agency for Healthcare Research and Quality, Rockville, MD. Statistical Brief \#331, 2011 (available at https://meps.ahrq.gov/data_files/ publications/st331/stat331.shtml, accessed June 20, 2020)

28. Stroup TS, Gray N. Management of common adverse effects of antipsychotic medications. World Psychiatry 2018; 17(3): 341-356
29. van Westrhenen R, Aitchison KJ, Ingelman-Sundberg $M$, Jukic M. Pharmacogenomics of Antidepressant and Antipsychotic Treatment: How Far Have We Got and Where Are We Going? Front in Psychiatry 2020; $12: 94$

30. Vilches S, Tuson M, Vieta E, Alvarez E, Espadaler J. Effectiveness of a Pharmacogenetic Tool at Improving Treatment Efficacy in Major Depressive Disorder: A Meta-Analysis of Three Clinical Studies. Pharmaceutics 2019; 11(9): 453

31. Villeneuve JP, Pichette V. Cytochrome P450 and liver diseases. Curr Drug Metab 2004; 5(3): 273-82

32. Whiteford HA, Degenhardt L, Rehm J, Baxter AJ, Ferrari $A$, Erskine HE, et al. The global burden of mental, neurological and substance use disorders: an analysis from the Global Burden of Disease Study 2010. PLoS One 2015; $10(2)$ : e0116820 (2015)

33. Whiteford, HA, Degenhardt, L, Rehm, J, Baxter AJ, Ferrari AJ, Erskine HE et al. Global burden of disease attributable to mental and substance use disorders: findings from the Global Burden of Disease Study 2010. The Lancet 2013; 382(9904): 1575-86

34. Wijesinghe R. A review of pharmacokinetic and pharmacodynamic interactions with antipsychotics. Ment. Health Clin 2016; 8,6(1): 21-27

35. Winner JC, Carhart JM, Altar CA, Goldfarb S, Allen JD, Lavezzari $G$, et al. Combinatorial pharmacogenomic guidance for psychiatric medications reduces overall pharmacy costs in a 1-year prospective evaluation. Curr Med Res Opin 2015; 31: 1633-1643

36. Wittchen HU, Jacobi F, Rehm J, Gustavsson A, Svensson $M$, Jonsson B, et al. The size and burden of mental disorders and other disorders of the brain in Europe 2010. Eur. Neuropsychopharmacol 2011; 21(9): 655-679

37. Yoshida K, Müller DJ. Pharmacogenetics of Antipsychotic Drug Treatment: Update and Clinical Implications. Mol Neuropsychiatry 2020; 5(Suppl 1):1-26

Correspondence:

Stefano Bramante, MD

Rita Levi Montalcini Department of Neurosciences, University of Turin

Via Verdi 8, 10124 Turin, Italy

E-mail: stefano.bramante@unito.it 